\title{
Observation End Day
}

National Cancer Institute

\section{Source}

National Cancer Institute. Observation End Day. NCI Thesaurus. Code C82569.

The final day of an observation. 